Journal of Universal Computer Science, vol. 18, no. 1 (2012), 62-85

submitted: 15/7/11, accepted: 9/12/11, appeared: 1/1/12 @ J.UCS

\title{
Accessible Lifelong Learning at Higher Education: Outcomes and Lessons Learned at two Different Pilot Sites in the EU4ALL Project
}

\author{
Jesus G. Boticario, Alejandro Rodriguez-Ascaso, Olga C. Santos \\ Emmanuelle Raffenne \\ (aDeNu Research Group, Artificial Intelligence Dept., Computer Science School, UNED \\ Madrid, Spain \\ jgb, arascaso, ocsantos, eraffenne@dia.uned.es) \\ Lydia Montandon \\ Atos GIBS (Global Innovation and Business Strategy), Madrid, Spain \\ lydia.montandon@atosresearch.eu) \\ David Roldán, Félix Buendía \\ (Universidad Politécnica de Valencia, Valencia, Spain \\ darolmar, fbuendia@upvnet.upv.es)
}

\begin{abstract}
The EU4ALL project (IST-FP6-034778) has developed a general framework to address the needs of accessible lifelong learning at Higher Education level consisting of several standards-based interoperable components integrated into an open web service architecture aimed at supporting adapted interaction to guarantee students' accessibility needs. Its flexibility has supported the project implementation at several sites with different settings and various learning management systems. Large-scale evaluations involving hundreds of users, considering diverse disability types, and key staff roles have allowed obtaining valuable lessons with respect to "how to adopt or enhance eLearning accessibility" at university. The project was evaluated at four higher education institutions, two of the largest in Europe and two mediumsized. In this paper, we focus on describing the implementation and main conclusions at the largest project evaluation site (UNED), which was involved in the project from the beginning, and thus, in the design process, and a medium-sized university that adopted the EU4ALL approach (UPV). This implies dealing with two well-known open source learning environments (i.e. dotLRN and Sakai), and considering a wide variety of stakeholders and requirements. Thus the results of this evaluation serve to illustrate the coverage of both the approach and developments.
\end{abstract}

Keywords: Educational standards, adaptive learning, user modelling, service oriented architecture, web accessibility, learning objects, metadata and learning, user-centred design Categories: H.1.2, H.3.5, H.4.2, H.5.2, H.5.4, J.7 


\section{Introduction}

Despite available legislation worldwide ${ }^{1}$ and expected benefits from student-centred approaches, students are not properly supported in their accessibility needs nor their individual preferences when they have to deal with administrative and educational procedures. In this sense, the European Higher Education Area [EHEA, 09] promotes the consideration of functional diversity issues (i.e. disabilities) in Higher Education (HE) from enrolment to assessment. Moreover, Technology Enhanced Learning (TEL) aims at improving students learning experience through interactive systems support, which ideally should deal with inclusive student-centred learning scenarios [EC-TEL, 10; Herrera, 09].

To develop inclusive TEL, two key issues, personalisation (i.e., user-centred adaptation) and accessibility must be addressed, and both are to be integrated in order to cope with students' personal needs while interacting with the Learning Management Systems (LMS) provided by most HE Institutions. Moreover, to make the approach interoperable and reusable, it should be based on standards and open software solutions.

Adaptive learning systems have undergone considerable changes over the last decade, especially thanks to web developments [De Bra, 08]. Initially there were research prototypes for developing adaptive learning environments [Brusilovsky, 03] but more recent efforts are focussed on providing general solutions focussed on extending existing educational standards to support adaptive course delivery addressing students' individual needs [Boticario, 08]. Adaptive learning has been addressed in a wide variety of European projects that consider standards and LMS [see Section 2], or more up-to-date service oriented architectures that can be integrated into the Web 2.0 and social learning approaches [Muñoz-Organero, 10]. However, personalisation, which covers adaptiveness and adaptability [Fink, 98], is a long-standing open issue and there is not currently any system that supports full adaptiveness.

In turn, there is vast literature on the many pending difficulties and barriers that hamper the educational needs in HE of a growing number of students who have functional diversity issues or require adaptations due to their age, mostly because adult students are the main target of the Lifelong Learning paradigm (LLL) [Dunn, 03; Seale, 06; May, 10]. Regarding those from disadvantaged groups, the European LLL program remarks that Information and Communication Technology (ICT) services should enable people with disabilities as well as elderly citizens enhance their potential and autonomy, thus helping them in embarking in new professional activities, and enjoying opportunities just as any regular citizen [EU-Riga, 06].

User centred standards offer guidance on accessibility, design for all and general usability. They are developed to improve the user experience when interacting with software. This type of standards is being legally enforced by legislative actions in many countries. However, as for the appropriate standards support in accessible and adaptive education is concerned, there are overlapping and contradictions between

\footnotetext{
${ }^{1}$ ADA in the US, Australian DDA, SENDA in the UK, BITV in Germany, LISMI and LIONDAU in Spain, Brazil Law on Accessibility, Disability Act in Ireland, The Stanca Act in Italy and The UN Convention on the Rights of Persons with Disabilities, among others.
} 
available standards to manage accessibility issues and dynamic support in terms of i) users' models, ii) learning scenarios, iii) interaction preferences, iv) devices capabilities, and v) metadata for specifying the delivery of any resource to meet users' needs [Moreno, 09].

In this context, the EU4ALL project (IST-FP6-034778) was funded by the European Commission to construct a general framework and extensible architecture of European-wide services that enable all students, including disabled students, or students with special needs, to access HE studies, from enrolment to examination and graduation. The project involved 15 partners from 8 European countries with varied roles in the consortium [see Section 3] and ended in March 2011, after almost 5 years of research and development. Four pilot sites were defined in the project: two largesized at UNED and UKOU and two small-sized at IPL and UPV. For the paper, we have chosen a representative of each kind, actually the largest and more comprehensive project evaluation site at UNED and the more challenging of the small pilot sites, which took place at UPV, involving an additional learning environment to those originally planned (i.e. Sakai). In particular, here we focus on describing the implementation and main conclusions at these two different sites. UNED (i.e., the National University for Distance Education in Spain) is a university that was involved in the design of the EU4ALL approach, and UPV (i.e. the Universidad Politécnica de Valencia) is a university that adopted the EU4ALL approach, which implies dealing with two different open source learning environments, dotLRN and Sakai, and considering a wide variety of stakeholders and requirements. Taking into account the variety of issues involved in these two sites the results of the evaluation described in the paper serve to illustrate the coverage of both the approach and developments.

The structure of this paper is as follows. First, an overview on the state of the art on accessible and adaptable learning is presented. Then, the EU4ALL project is described, including an overview of objectives, architecture, end user services, and some methodological issues. After that, the results from the EU4ALL approach implementation in two pilots is reported, namely UNED and UPV, focusing on the evaluation processes. Next, the final outcomes and lessons learnt along with future work are put forward.

\section{Accessible and adaptable learning: State of the Art}

There exist many LMS available in the e-learning context [Edutools], and most universities use LMS to support their teaching and learning process online [MuñozMerino, 09]. LMS facilitate the management of the learning process (but do no control it), and usually offer the same solution to each student, no matter the different goals, needs and background students may have. Hence, fully adaptiveness is not reached in LMS, which still provide little support for adaptation [Hauger, 07].

LMS are to address the need for personalisation and inclusive support in the learning process, something which is widely required [e.g. Lanzilotti, 06; Seale, 08, Sawyer, 08].

Adaptive learning systems and LMS developments have been disconnected for a long time. Nevertheless, some initiatives recently started to bridge the gap between these two worlds. Researchers are extending user profiles of existing LMS for user modelling and introducing personalisation functionalities into different LMS modules 
[Raffenne, 09]. Likewise, researchers on adaptive learning systems are finding ways to integrate their adaptive learning systems within existing LMS. Efforts in this direction are found mostly in European research projects, such as the aLFanet [Boticario, 07], ALS [Ghali, 08], GRAPPLE [de Bra, 10] and FLEXO [Flexo]. They aim to consider research efforts coming from previous projects, such as OPAL [Conlan, 02], OLO [Rodríguez, 02] and KOD [Karagiannidis, 00].

In this context, service oriented architectures have been suggested to cope with the required development issues, which require using interoperability specifications such as the Web Services for Remote Portlets [WSRP], an OASIS-approved networking standard protocol. In the context of learning systems, the IMS Basic Learning Tools Interoperability [IMS-BLTI] specification is being developed to allow remote tools and content to be integrated into a LMS. Examples of service oriented architectures are the e-Framework for Education and Research [e-Framework] that provides information to institutions on investing in and using information technology infrastructure, the Open Knowledge Initiative [OKI], which develops specifications that describe how the components of a software environment communicate with each other and with other enterprise systems and promotes sustainable interoperability and integration by defining standards for service oriented architectures in terms of Open Service Interface Definitions (OSIDs), and the Fluid Project [Fluid] which designs user interfaces, builds web tools, teaches inclusive design and integrates interface components into open source applications. Moreover, the IEEE Learning Technology Systems Architecture [LTSA], which is part of the IEEE Learning Technology Standards Committee, focuses on defining a framework of reference at architectural level for educational systems that is neutral at pedagogical, content, cultural and implementation levels.

There have been several research efforts to develop inclusive learning systems that go further than complying with the WCAG, offering generic pedagogical tools that consider some kind of adaptation [Seale, 10]. A wide range of projects have focused on accessibility and disability issues. Through Assistive Technology to Employment [Beyer, 07] produced health and safety training materials for those supporting people with learning difficulties in employment. Benchmarking tools for the Web [BenToWeb] provided a mixture of advanced developments in the area Webbenchmarking, quality assurance and compliance (with accessibility requirements). Multimodal Collaboration Environment for Inclusion of Visually Impaired Children [MICOLE] developed a system that supports collaboration, data exploration, communication and creativity of visually impaired and sighted children. The CORES project [Cudd, 94] incorporated user modelling to support the required degree of flexibility and intelligence to dynamically adapt the user interface to the users' needs regarding speech characteristics. The IRIS project (IST-2000-26211) defined a combined user and device model to be used for content negotiation. Moreover, legal, political and socio-economic considerations are considered at the HEAG (HE Accessibility Guidelines) [HEAG], the European Agency for the Development of Special Needs Education.

Unfortunately, all these efforts are still in the research arena. Thus, current LMS do not properly cover personalisation and accessibility issues and they are still struggling to support the reusability requirements coming from the need of a pervasive usage of standards. Almost none of the existing LMS supports a wide range 
of educational standards [Santos, 07], but most of them only offer the possibility of uploading courses with Sharable Content Object Reference Model [SCORM]. There are also challenges related to the usage of educational standards to provide adaptations through the entire life cycle of e-learning [Van Rosmalen, 04] as well as to provide a holistic approach for accessible e-learning [Phipps, 06].

In summary, HE should be an accessible service for all to consider the specific needs of each student and to adapt their processes based on the context, environment, devices, competences, skills and individual abilities [Moreno, 09].

\section{EU4ALL project}

The EU4ALL project (European Unified Approach for Accessible Lifelong Learning, IST-2005-034778) is a European project under the 6th Framework Program, within the FP6-2005-IST-2.5.11 eInclusion research objective. Although in the e-Inclusion area, it fully focused on technology applied to education. In particular, it addressed the needs of Accessible Lifelong Learning at HE. EU4ALL is a 48-months (finally extended to 54, from October 2006 to March 2011) integrated project which involves major stakeholders on both the demand and supply sides.

This project was ambitious from the outset, with a wide variety of interrelated scientific and technical issues involved. To name but a few aspects, which have significantly influenced the project outcomes: i) the complexity of developing a conceptual and operational users' requirements framework, ii) the evolving nature of standards, iii) the transversal issues required in developments, and iv) the inclusion of new partners, third-party tools and a new LMS in the last stage of the project lifecycle.

The partnership covers the whole value chain for the provision of e-learning accessible services, from research, to development, project results use and exploitation. The consortium is composed of 15 partners from 8 different European countries, including mega-universities (the Open University based in the United Kingdom and UNED in Spain), medium and small size universities (University of York in the United Kingdom, Instituto Politécnico de Leiria in Portugal and UPV in Spain) and the European Association of Distance Teaching Universities -EADTU (covering over 200 universities and around three million students across Europe), along with leading IT companies (Atos Origin and Indra Software Labs in Spain, eXact Learning Solutions in Italy and Tribal in the United Kingdom), institutes of applied research and development (Fraunhofer Institute of Technology in Germany), research organizations (Centre for Social Innovation - ZSI in Austria and Centro Interuniversitario di Ricerca per lo Sviluppo Sostenible - CIRPS in Italy) and organization of users (DPI Italia and Information Society Open to Impairments eISOTIS in Greece). Partners played different roles in the project. The University of York coordinated the user requirements elicitation and the evaluation methodologies. Under the scientific coordination of UNED, the Fraunhofer Institute, Atos Origin, Indra Software Labs and UNED carried out the technical developments, from architecture design to specific components implementation. eXact Learning improved the accessibility of their ePortfolio and Tribal designed the Guidance4all component as well as most of the training materials. ZSI was in charge of studying the socioeconomical context, the market needs and the legal framework. CIRPS, DPI Italia, e- 
ISOTIS and EADTU dealt mainly with the dissemination of the project, in particular to the general public and to HE institutions. UNED, the Open University, Leiria Technical Institute and Valencia Technical University performed the project evaluation activities. Finally Atos Origin was in charge of the overall project coordination.

\subsection{EU4ALL objectives}

The main goal of EU4ALL was to enable all students, including disabled students, or students with special needs, to access HE studies, from enrolment to examination and graduation. Technically, EU4ALL aimed at researching and developing a general open framework consisting of several standards-based interoperable components integrated into an open web service architecture to support the development of accessible and personalised learning services in the context of $\mathrm{HE}$ that provide adapted interaction to guarantee students' accessibility needs at HE [Santos, 11a; Douce, 10]. The framework was designed based on a substantial number of face-toface stakeholder interviews and an on-going work within the e-learning and accessibility standards arena. The focus of the framework was to establish a high level view of accessibility provision at $\mathrm{HE}$ institutions from a technical perspective.

The specific objectives of EU4ALL were as follows [Boticario, 06]: (1) Achieve a unified and usable vision on standards, users' requirements, service definition, technologies, (2) Implement in terms of standards an open and extensible architecture of services for accessible LLL supporting students and service providers, (3) Provide user-centred services that consider individual user's needs and preferences, pedagogical guidelines and adaptive behaviour, (4) Bring together major service providers (e.g., Open University, UNED, EADTU) to foster the awareness of best practices in providing educational services for accessible lifelong learning, (5) Impact on major standardisation bodies from project developments, and (6) Create a channel for the diffusion and benchmarking of this research across Europe.

As it is described in the rest of this paper, the project has obtained valuable outcomes according to these objectives, specifically on defining a body of knowledge on user requirements, standards consensus from the most up-to-date standards available, interoperability of the architecture components, flexibility and applicability of developments in large- medium- and small-size HE institutions, involvement of major stakeholders, and dissemination and use of knowledge from project outcomes.

\subsection{Standards-based components and the architecture}

In line with the aforementioned objectives, the EU4ALL architecture was built with openness and standard compliance in mind. Taking into account the existing overlaps regarding standards, a consensus was reached in the project on the integration of the different standards to facilitate the mediation process between contents and users' interaction preferences, selecting ISO Digital Resource Description and ISO Personal Needs and Preferences (i.e. ISO/IE 24751:3 and ISO/IE 24751:2) and providing the appropriate binding for them [ISO, 09]. The selection of these standards was done taking into account the experience of experts from the EU4ALL consortium that were involved in standardisation bodies (ISO, IMS, W3C). 
The Service Oriented Architecture (SOA) paradigm is the base of the design of the EU4ALL architectural framework, as it guarantees the flexibility and loose coupling between the components, allowing the addition of new services to the framework as they become available. The EU4ALL framework provides specifications for the web service interface of each component: the User Model -UM (implemented at UNED by the aDeNu group), the Recommender System - RS (implemented at UNED by the aDeNu group), the Metadata Repository - MR (implemented by ATOS) and the Content Personalisation - CP (implemented by INDRA).

The User Model (UM) stores and manages user information related to: i) demographics (personal information), ii) preferences (general and accessibility preferences), iii) learning styles (in terms of Felder dimensions [Felder, 88]), iv) goals and competencies, v) progress (qualifications, certificates and licenses obtained by the student), and vi) psychological profile (indicators about attention, memory, time management and other skills). UM implements the IMS-LIP information model and binding to represent the aforementioned information, and a XML binding of the ISO/IEC-24751-2 (ISO PNP) “Access for All personal needs and preferences for digital delivery” to represent accessibility preferences. Its web service interface allows for communication with other components of the framework, such as the RS and the CP.

The Recommender System (RS) is meant to support users while interacting with the LMS by highlighting appropriate actions to be carried out. For this, the concept of Semantic Educational Recommender Systems (SERS) has been proposed [Santos, 11b], which helps dealing with educational issues that have to be identified by educators. The TORMES methodology was defined to support this approach, which is based on the ISO standard 9241-210 and aims to involve educators in the process of designing educationally oriented recommendations through user centred design methods. These are of particular importance when guiding users with disabilities [Santos, 10].

The Metadata Repository (MR) is in charge of storing and managing metadata of learning objects. Several types of metadata are considered and the corresponding standards are used for representing them: a) IEEE 1484.12.1 Learning Object Metadata [LOM] with general information about the resource, b) ISO/IEC 24751-3 (ISO-DRD) “Access for all” digital resource description with information regarding accessibility of the resource, and c) ISO/IEC 15938 "Multimedia content description interface” (MPEG-7) with general information about multimedia resources. The metadata is formatted in XML and stored in a XML database (eXist).

The Content Personalisation (CP) is responsible for selecting the resource that best suits users' needs and preferences. It relies on the UM and MR to gather the data regarding user preference and resource description and to perform the matching between the two for selecting the best resource. User and resource data are translated into an intermediate language, and processed by an inference engine. The CP offers a web service interface with two operations: one for selecting a resource given a user's request, and the other to check the availability of accessible(s) alternative(s) for one or several users and one or several resources.

In all, with the rest of the architecture components (Device Model, eService Server...) 16 standards and specifications have been considered (ADL: SCORM; IMS: 
LIP, accLIP, LD, QTI; ISO: 24751, 9999; IEEE: LOM; W3C: UaProf, DCO, DCCI, DDR, DPE, CC/PP, SOAP, WSDL; Other: WURFL) [Boticario, 11].

\subsection{End user services}

In this section, the end user services implemented in UNED and UPV pilot sites are defined. The coverage of UNED's pilot site has been the largest in the project both in terms of the number of users involved in the evaluation and the variety of implemented services. In turn UPV, which involved an additional LMS to integrate into the architecture, provided the requirements of a medium-sized university. Therefore, the services described here illustrate the feasibility of the whole framework and the architecture.

At the time of implementing the EU4ALL end user services, each pilot site may need to select the appropriate ones from the general portfolio of EU4ALL services, as well as to slightly adapt them in order to suit the particular needs of the organisation to run the service [Santos, 11c].

Several kinds of stakeholders are involved in the delivery of the services, namely: a) Lecturers, who are supported in producing accessible materials and are provided with tools for supervising course's accessibility before it is offered to students; b) Students, who are allowed to express their accessibility needs through the system as well as to provide their feedback on system's behaviour; c) Disability officers, who support students by assessing their needs, and serve as a liaison between students and other university professionals to address any problems caused by inaccessibility of activities or materials; d) Transformation officers, who work on the adaptation of materials, in co-ordination with lecturers and librarians; and e) Librarians that support the tagging and management of learning materials in electronic repositories.

Taking into account the stakeholders involved, the end user services instantiated at the pilot sites are as follows:

- Needs Assessment Service (NAS). It allows users to complete and/or check the information stored by the system about their accessibility needs and preferences, as well as their psycho-educational style and needs (complies with ISO/IEC 24751 and IMS Learner Information Package version 1.0.1). This end user service is complemented with a face to face assessment of user needs in order to ensure the applicability of the preferences identified.

- Authoring Support (AS). It aims to support authors in creating accessible materials, including those with special notations (e.g., Maths through MathMLbased content). The eXe open source authoring tool [eXe] has been used (and extended to cope with accessible math notation) because it assists teachers in publishing web content, supports templates to deal with accessibility issues and its outcomes can be exported into standards (IMS Content Package, SCORM 1.2, IMS Common Cartridge).

- Course Accessibility Information Service (ACCINFO). It aims at a holistic accessibility assessment of a course taking into account the needs and requirements of an individual student and all the course constituents that may have an impact on its overall accessibility, including digital resources, [see RESACCINFO below], equipment requirements, learning activities, and assessments. This service is consulted by a student to assist with decisions 
regarding whether to enrol in a course, or by a professional in order to identify and handle accessibility issues on behalf of enrolled students.

- Resource Accessibility Information Service (RESACCINFO). It checks whether a media item is accessible to a student based on the given user profile. It is invoked by ACCINFO or by a professional in order to retrieve the accessibility status of the media for a given student. An accessibility assessment based on a previously defined profile is also supported.

- Resource Adaptation Management Service (RESADAPT). It mediates the manual adaptation of an inaccessible resource through a management workflow tailored to conditions found at a respective institution. Firstly, it allows a request to be made for the transformation of an inaccessible resource by either a professional or a student. The request for the resource transformation is sent to those departments within an organisation where the provision of the accessible resource is managed. Content transformation requests are intended to happen preemptively of a course running once student preferences are available. It is either invoked by a professional in order to capture issues raised by ACCINFO, automatically by the $\mathrm{CP}$ when no appropriate adaptations were found or requested indirectly by a student experiencing difficulties through the feedback service.

- Feedback Service (FEEDBACK). It provides a mean to create surveys collecting data about the accessibility issues experience by students while accessing learning materials. This is performed in a structured, controlled way while offering for an adaptive, multi-modal delivery.

Next, we comment on the methodology used along the project. After that, we detail how the EU4ALL approach was used in practice in the two universities.

\subsection{Methodology}

The EU4ALL project has set up methodologies on various critical issues as for accessibility at $\mathrm{HE}$ is concerned, which are to be taking care in further exploitation activities. Firstly, a scenario based approach [Carroll, 00] has been followed to produce a comprehensive set of requirements for institutions that are willing to provide accessible services [Petrie, 09]. Further, the methodology also assesses the level of institution preparedness regarding accessibility practice, which depends mainly on social, political, organisational and management issues and which allows to establish the appropriate exploitation strategy for each level [Weiermair-Märki, 09]. Secondly, it has defined an open service-oriented approach, which matches current cloud computing methodologies drawing on standards-based services that can be integrated into current (dotLRN, Moodle, Sakai) and future LMS and Personal Learning Environments (PLE) to support accessible interaction to students [Santos, 11c]. Thirdly, it has set up a clear user centred evaluation approach and methodologies on how to develop and evaluate services on supporting accessible elearning at HE [Weiermair-Märki, 09]. Regarding accessibility evaluation methodologies and end users' involvement, a central issue has been to guarantee end user participation in the development of end user services in terms of the production life-cycle, i.e. update and evolve architecture services according to service validation cycle [Fernández del Viso, 11]. The iterative design cycle was a direct consequence of each validation and evaluation process. 
The evaluation measured the framework services from two main viewpoints, namely the user experience and the framework adoption aspects. User experience aspects have to do with the accessibility and usability of services: Needs Assessment Service (NAS) accuracy, optimal strategy for personalising learning contents with Content Personalisation (CP), appropriateness and suitability of the recommendations generated by the Recommender System (RS), or availability and usefulness of the Feedback Service (FEEDBACK). Framework adoption aspects deal with the benefits and disadvantages derived from the application of EU4ALL in the university, the usefulness of the information collected and processed by the provided services, and the ease of integrating these services into the current and planned university's systems. Moreover, results from the validations and evaluations were analysed and fed back to developments to allow improvements and refinements of the architecture and services.

\section{EU4ALL in practice}

User requirements elicited in EU4ALL have fed the design process of the framework and its end user services. Several methods of user requirements elicitation were used within the project. On the one hand, analysis of email discussion lists (e.g. Dis-forum, SpLD forum and dyslexia forum from JISC in the United Kingdom and available resources worldwide were carried out [Petrie, 09]. On the other hand, several elicitation methods from user centred approaches were followed. In particular, in Spain the following activities were performed: 8 interviews, 1 focus group, and 188 online surveys, 55 among professionals and 133 among students.

This elicitation work, and the subsequent analysis, produced a list of user requirement propositions, grouped in different themes (e.g., adapted contents and delivery, assistive technologies, communication, LMS, libraries, course/modules, support services, institutions, and assessments). The project has not addressed all these propositions, the reasons being either that not all the propositions are applicable to all countries/institutions with the same level of priority, some of them were not within project's objectives, limitations in time and budget, etc. The addressed propositions led to the definition of the end user services introduced above.

These services were instantiated and evaluated with students and professionals in different pilot sites. In particular, at UNED 61 students and 62 professionals were involved. In turn, at UPV, 10 students and 6 professionals used the services. Next we detail the implementation of the EU4ALL approach at two significant project sites: 1) UNED, a large university, which was involved in the design of the approach from the outset, and 2) UPV, a medium-sized university, which join the project in the last year and adopted the approach.

\subsection{Implementation at a large university}

At UNED, EU4ALL researchers have closely collaborated with the University's Disability Office (UNIDIS) along the whole project's life. Through this collaboration, links have been established with relevant University's units playing a role in the provision of support to students with disabilities. Furthermore, communication with UNED's students with disabilities has been mediated through the on-line community 
for Attention to Disability, which was formally set up in March 2007 by UNIDIS and supported by UNED's project team [Rodriguez-Ascaso, 08]. This community has been used to recruit users for both user requirements elicitation and large pilot site evaluation. Also, it has been used for project's dissemination purposes. UNIDIS, together with other relevant university departments, has also taken part in the evaluation of the large pilot site, which is described in more depth below. Regarding the LMS, UNED uses dotLRN for the whole range of university studies and currently there are over 200,000 users registered [Santos, 07].

\subsubsection{Technical issues}

The LMS used at UNED is based on OpenACS/dotLRN. OpenACS is a toolkit for building scalable, community-oriented web applications. OpenACS provides core services whereas dotLRN is a set of tools built on OpenACS to provide e-learning services (learning objects delivery, communication tools, assessment tools, etc.). dotLRN also provides a portal through which users can access their courses and the related tools.

To integrate the EU4ALL framework with dotLRN, UNED has implemented clients for each of the EU4ALL components that provide services to the LMS [Raffenne, 09]. The clients provide interfaces between the EU4ALL components and dotLRN main services [see Fig. 1].

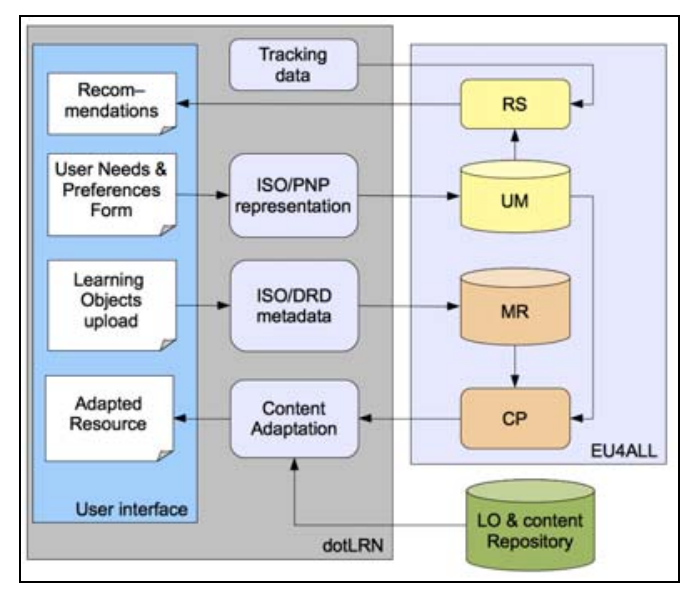

Figure 1: overview of the integration with $\operatorname{dotLRN}$

The UM client provides a form to explicit preferences regarding accessibility to learning contents [see Fig. 2]. Users' preferences are forwarded to the UM for their storage. In the same way, when learning objects are uploaded to the LMS, such as SCORM or IMS-LD courses, the ISO/DRD metadata included in the UM are sent to the MR.

The RS client provides an interface to display recommendations for the current user [Santos, 11b], either in the user portal or the course one. When the user accesses 
a portal in which it has been included, the client requests recommendations from the RS, parses the response and formats it in HTML for its display.

Finally the CP client provides the personalization service when requesting learning content. When a user requests a document containing resources that may require adaptation, a request is sent to the $\mathrm{CP}$ to obtain the identifiers (URI) of the alternative resources. The CP communicates with the UM and MR. If any alternative resource exists for that content and user, the document is modified to replace the original resource with its adaptation.

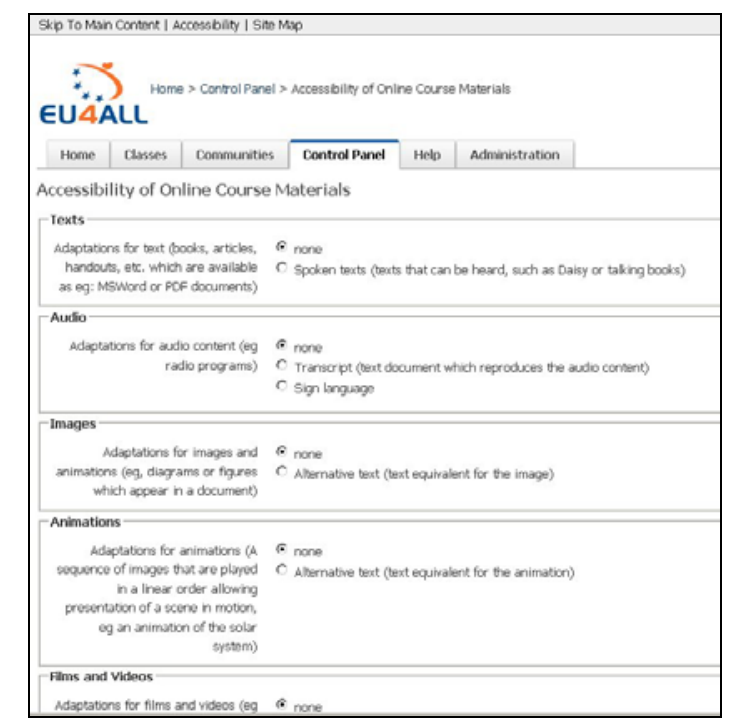

Figure 2: Form added in dotLRN to gather accessibility preferences

\subsubsection{End user services evaluation}

The objective was to evaluate the EU4ALL services [see Section 3.3] implemented in the dotLRN prototype at the UNED pilot site, namely: the Needs Assessment Service (NAS), the Resource Accessibility Information Service (RESACCINFO), the Resource Adaptation Management Service (RESADAPT), and the Feedback Service (FEEDBACK). Moreover, the following components were also evaluated from the end users viewpoint: the Content Personalisation (CP) system to access the content of a course according to users' accessibility preferences as expressed by the NAS, and the Recommender System (RS), giving students guidance on how to improve their learning experience.

A total of 123 people (61 students and 62 professionals) took part in the evaluation activities of the UNED pilot site: three workshops (53 participants), two focus groups (9 participants), face-to-face evaluation sessions (9 participants), and remote evaluation sessions (52 participants). Students with visual, hearing or physical impairments took part in the evaluation. They declared to use the following accommodations when studying through the Internet: hearing aid (6), enlarged text and hearing aid (1), enlarged text (5), screen reader (9), screen magnifier (5), screen 
magnifier with text-to-speech technology (5), large monitor screen (2), voice recognition (2), voice recognition and adapted mouse (2), adapted keyboard (2). 22 students declared to use no accommodations. In turn, the distribution of the roles of the 62 professionals who took part in the evaluation at UNED is as follows: lecturers (11), disability unit officers (11), technical area staff (5), librarians (5), content and multimedia officers (11), staff from external entities providing accessibility related services (7), members of standards organisations (2), and staff from other university departments (10).

On-line materials were created for being used by students and professionals as part of the services under evaluation, such as two forms for the NAS service. Moreover, two SCORM courses were created, one on basic maths and one on job search. These courses included different media items (text -including mathematical content, images, audio, and video) together with three adaptations: i) text description for images; ii) captions, transcripts and sign language interpretation for the audio; and iii) transcripts for videos. A DAISY book was created as an alternative to the job search SCORM course. Also, two online questionnaires were produced to be used within the FEEDBACK service. Furthermore, a set of recommendations were elicited with the TORMES methodology and instantiated in the LMS to be displayed when the design conditions apply.

During the workshops and focus groups, end user services were presented to groups of potential users. After the services were presented, their most relevant characteristics were discussed with participants by following a topic schedule. Qualitative information was obtained about the willingness to use the specific services and to adopt the overall framework.

Remote and face to face evaluations provided both subjective and objective indicators on the user experience of EU4ALL services.

As for the accessibility and usability of the services, positive results were obtained, both from objective and subjective measurements, for almost all user groups. The user group for which poorer results were obtained is the one of the people who used both screen magnifying and screen reading software. To illustrate this, Figure 3 represents the time it took students to carry out task 1 (i.e. fill-in the personal preferences regarding the accessibility to electronic learning materials), presented by functional profile.

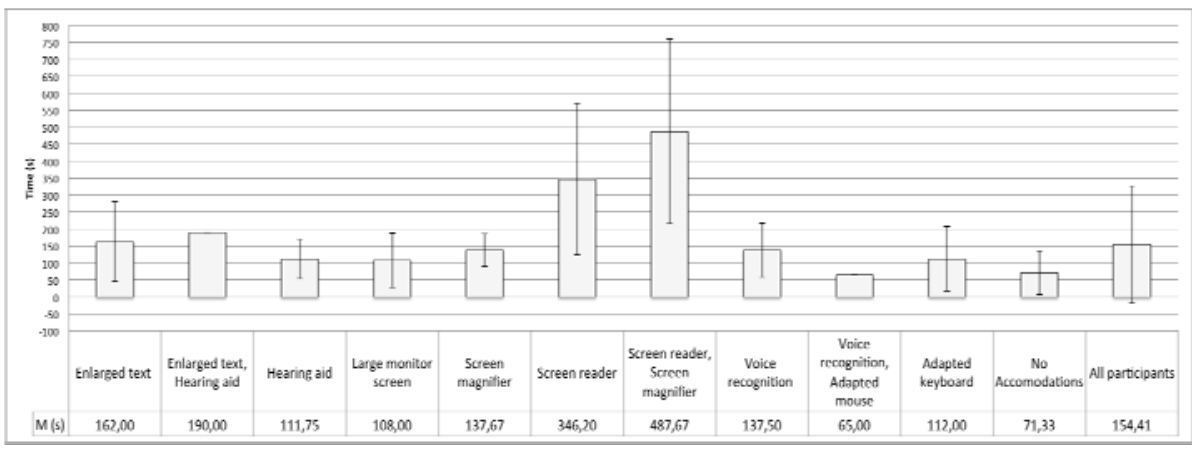

Figure 3: Time for filling-in accessibility preferences, by functional profile 
Regarding the accessibility of basic contents, such as video or mathematical text content, it is still a challenge, even if they have been created with accessibility in mind, because of limitations in accessibility characteristics of players and user agents, respectively.

Regarding the NAS, no mismatches were identified among the accessibility preferences stored by the system and the ones actually used by participants. However, a greater involvement of UNED disability officers with the provision of the service will be needed in order to give support to students, as well as to control and monitor the resources UNED commits to provide. Furthermore, non-exclusive adaptations need to be offered for adapting learning objects, and additional parameters need to be considered, including the type of the subject or the object's file format.

The RESACCINFO has been considered as very helpful by lecturers. However, usability of RESACCINFO service has to be improved, according to their opinion.

The RESADAPT service has been validated successfully with lecturers, librarians and media production officers, both from UNED and from external organisations.

As for the FEEDBACK service, it needs to be improved. Evaluation has shown that users need more system assistance to provide information that can be really used by professionals to fix accessibility problems.

The Recommender System seems to be a valuable service to users, although the evaluation context at UNED pilot was not the most suitable to explore real advantages of such service. In any case, its value has been demonstrated in other scenario [Santos, 11b].

In the case of the Content Personalisation, students are satisfied with the way it provides access to learning contents according to each student's preferences. However, students seem to prefer the system to offer all available adaptations, as this gives them the possibility to choose among them [see Figure 4].

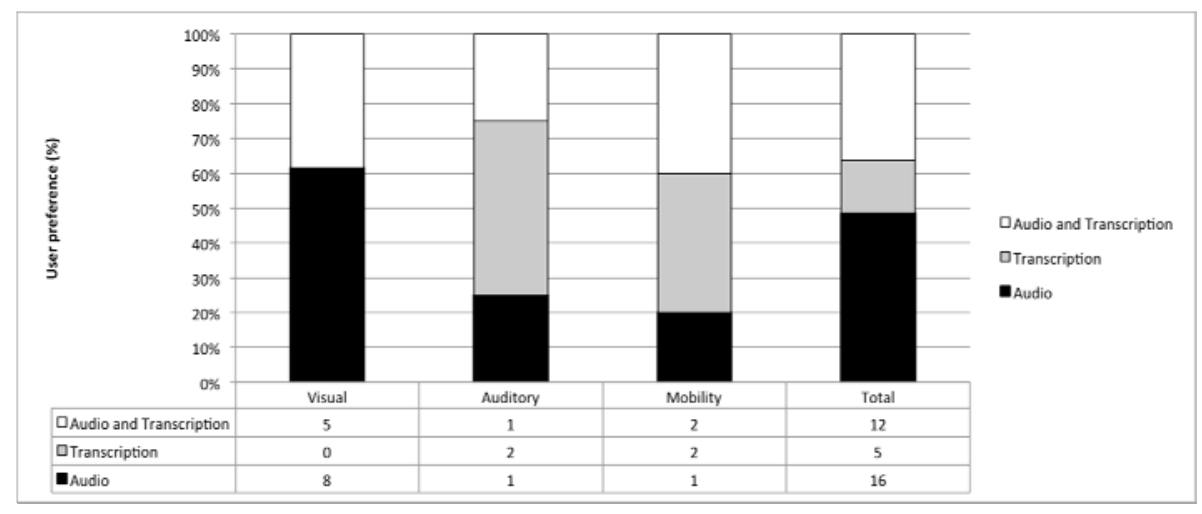

Figure 4: Participants' choices about their adaptation preferences according to their impairment type

\subsection{Implementation at a medium size university}

At the beginning of the year 2010, the UPV was invited to collaborate within the EU4ALL project. The main goal in this context was to adapt the Poliformat platform 
[Buendia, 09], used as the institutional LMS at UPV to integrate it within the EU4ALL framework. Poliformat is a product elaborated by the UPV technical staff and has been offered to students since 2005. It is based on the Sakai environment [White, 05]. This e-learning platform is being used every year by about 40,000 students and 2,600 members of teaching and research staff, in a hundred of undergraduate and Master degrees.

The implementation of the UPV pilot site was bounded to a reduced set of services provided by the EU4ALL framework since this university was incorporated in the project for the last year. Moreover, the evaluation of these services was implemented at the last phase of the project and this circumstance imposed a tight deadline in the experiences that checked the developed services at the UPV pilot site addressed to a small number of users who participated in these evaluation experiences. UPV staff team focused its actions in two main end user services: the assessment of needs (NAS) and the authoring and resource management services (AS and RESACCINFO).

Once this adaptation at the UPV pilot site was performed, a set of evaluation experiences were implemented in several UPV courses. This evaluation was assisted by the CEDAT foundation, which is the UPV disability office at the UPV in charge of assisting both disabled people who study and work at the university and those who are foreign to it. CEDAT contacted with the students who were involved in the evaluation of the UPV pilot site and assisted on the implementation of the assessment experiences. Next sections describe the technical details about the implementation of EU4ALL issues at UPV and the evaluation experiences developed in this context.

\subsubsection{Technical issues}

As aforementioned, Poliformat is based on the Sakai framework in which UPV became a developer partner since 2005. Sakai is a consortium of universities, colleges and commercial affiliates working in open partnership with standards organizations and other open-source initiatives to develop "community-source enterprise-scale software applications to enhance collaboration, research and teaching within HE” [White, 05]. The Sakai framework is based on an extensible service-oriented architecture for building and deploying enterprise-scale collaboration, teaching and research tools and services. Sakai framework, thus, relies on components providing features (services) and these services can be use to build Sakai tools [Roldán, 11]. Tools are units of software that generate a user interface.

Thus, Sakai Framework components can be easily extended to improve or modify current components features [see Fig. 5]. In order to integrate the EU4ALL approach in Poliformat (i.e. Sakai) the first modification was to extend PreferencesServices, which is the service of the framework in charge of managing User Preferences, so that it can connect to EU4ALL components (in this case, the User Modelling) through a new developed web services tier. At the end user side, a new tab into the Sakai preferences tool was also added to handle accessibility information, as in dotLRN.

The UPV technical staff team also modified Resources tool, which is one of the main Sakai tools as it can provide helper services to other tools (assignments, test \& quizzes, announcements, etc.) so that instructors can specify accessibility constraints for the resources they are about to upload to the site content area and students can only see resources matching their accessibility preferences. In this case, a first action 
was to incorporate in the Sakai Resource tool a new form to allow instructors input content attributes. These attributes were related to accessibility issues such as alternative media for text, audio or image contents, and their values were stored in the MR component. In a second step, the Resource tool was connected to the CP component in order to register the content alternatives depending on the user accessibility preferences.

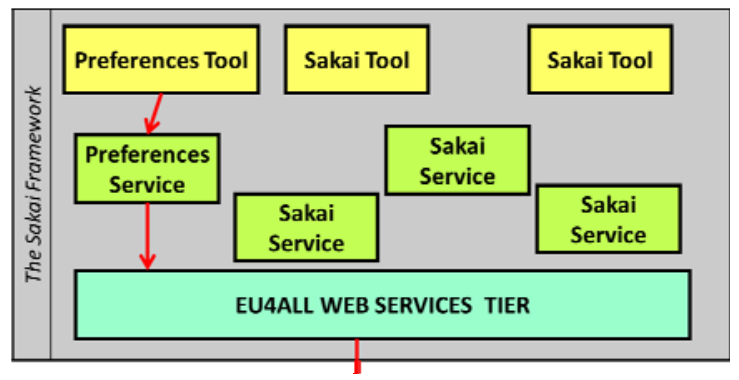

Figure 5: Sakai Framework: Tools \& Services

\subsubsection{End user services evaluation}

The evaluation of the experiences developed in the context of the UPV pilot site was based on a methodology mainly addressed to check the user's point of view. In this case, the UPV research team adopted a method based on gathering qualitative information about the user experiences and considering several phases in the evaluation process, from the assessment of the user profiles (either students or lecturers) to the final collection of their perspectives and their analysis.

The first phase consisted in defining specific learning scenarios in which these experiences were developed, establishing the items to be evaluated along the target experiences. This definition was supported by preliminary interviews with students and lectures. The second step was the specification of several research questions to check two main end user services in the EU4ALL contexts. These research questions were quite similar to those addressed by the UNED evaluation but they were bounded to a reduced set of end user services. The last phase was the collection of evaluation results from users who participated in the EU4ALL experiences through questionnaires submitted to end users either students or lecturers. Additionally, some informal interviews with UPV managers were implemented in order to obtain their view on the potential integration of EU4ALL services in the Poliformat platform [Buendia, 11].

The participants in the evaluation process were classified in two main groups: i) lecturers who provided course materials to be adapted in the EU4ALL context and ii) disabled students who were registered in different UPV courses, mainly in Computer, Business and Engineering disciplines. One of the main problems in the evaluation of the UPV pilot site was the diversity and heterogeneity of student profiles and courses. About ten lecturers were asked for participating in the project but only six of them were able to evaluate the UPV pilot site. In the case of students, CEDAT as the disability office at the UPV contacted with about 14 users and finally, ten students 
(four with hearing impairment and six with visual impairment) participated in the evaluation.

The evaluation was focused on two main areas: i) Computing courses and ii) Business courses. These courses were face-to-face (classroom-based) and they provided a set of instructional materials mainly based on text documents (PDF in most cases), and Microsoft PowerPoint presentations. However, they also included video or other multimedia formats. For instance, in Computing courses, audio versions were produced for pdf documents for describing lab instructions or adding additional information in graphical presentations (e.g. wiring diagram), which were difficult to read for visually impaired people. In the case of Business courses, some deployed materials were screencasts, lecture recordings and podcasts, which were captioned or provided transcripts to students with hearing troubles.

Students who participated in the evaluation experience tested the different courses they were assigned during two weeks and after this test, they were invited to fulfil a questionnaire related to check the NAS. In a similar way, lecturers who were involved in the project checked the introduction and review of the adapted resources. As aforementioned, 10 students participated in the evaluation process and they answered the questionnaire. Some quality criteria were defined to link these answers with the addressed research questions. For instance, the Handiness to evaluate if students had found easy the way to enter the accessibility preferences in the UPV pilot site or the Accuracy that referred if students considered that the preference options displayed the required features in a precise way. After the questionnaire submission a set of results were obtained in order to evaluate the student point of view about some NAS issues. These results were measured in a 1-5 scale, where the lower was the value the worse was the user satisfaction. In general, students were satisfied with the pilot tool that implemented the NAS, and in particular, issues related to the time required to fill the user preferences (Timeliness) or the tool Handiness were well valuated.

The next step was the connection of the questionnaire results with research about the User Experience such as "Are clear the questions about user needs or preferences?" or "Are these needs appropriately assessed?" that could be easily connected with the Understandabilty and Accuracy evaluation criteria checked in the questionnaire. The evaluation of research questions related to the Framework adoption such as "What are the benefits and drawbacks of the service?" could be checked by means of the Usefulness and Performance criteria. For instance, 60\% of students agree or strongly agree with the framework Usefulness and this percentage was $80 \%$ in the Performance item. Therefore, a relatively high benefit can be determined from these values. The research question about the "Integration with planned/current UPV systems "was only evaluated in an informal way by contacting with some manager responsible who stated their interest in incorporating the NAS within the Info-Accessibility initiative at the UPV.

Regarding research questions about Authoring and Resource Management services (i.e. AS and RESACCINFO), they obtained a neutral valuation. For example, the question “Are accessibility options enough for authors?" was evaluated by the Completeness item in which near a third of lecturers manifested a positive point of view. The question “Are they guided and effectively supported?” can be assigned to the Easiness (50\% agree or strongly agree) and Operability criteria (33\% agree). The assessment of "the benefits and drawbacks of the service" showed that the point of 
view of lecturers was balanced between the benefits and drawbacks provided by these services.

\subsection{Validation of the EU4ALL Approach}

The conclusions obtained from the evaluation at UNED's pilot site indicate firstly that, regarding framework adoption, EU4ALL's SOA constitutes a roadmap to improve institution's level of accessibility and therefore to comply with current Spanish legislation. Services were highly valued and provided a major progress comparing to those that are currently provided at the University, because EU4ALL services cover the learning process itself (e.g. adaptation facilities of contents through $\mathrm{CP}$ ) and they are not just bounded to enrollment and examination processes, which is the current focus at UNED. However, the project's technological outcomes still need to go through a technology transfer process where active participation of management and technical staff is absolutely crucial. Also, UNED needs first to improve basic accessibility problems that were not even part of project's objectives (e.g., ensuring that lecturers produce accessible MSWord, OpenOffice or PDF documents before they share them with their students). Therefore, awareness raising tools and mechanisms would be very useful to promote the creation of accessible documents.

In the case of UPV, the point of view of students was globally positive since they considered the suitability of the developed tools at the UPV pilot site to provide them accessible versions of instructional contents. Maybe the number of students who participated in the evaluation experience was low but it represented a significant sample within the disabled population at UPV. Moreover, informal interviews with other non-disabled students about the use of this pilot site confirmed this perception. On the other hand, the point of view of lecturers was tested using a questionnaire focused on the Authoring Tool service. This questionnaire was complemented with lecturer's interviews in order to get a more detailed view. In general, lecturers' comments were sceptic about the efficacy of the content Authoring service and they reported that a big effort developing adapted resources and researching about the generation of accessible contents was required. Therefore, new evaluation experiences should be implemented to obtain a more significant valuation by content developers.

\section{Conclusions: Outcomes, Leasons learnt and Future Work}

EU4ALL implemented a standards-based open and extensible architecture of services to provide accessibility at HE supporting students and service providers. The project developed a general infrastructure, which consists of several standards-based interoperable components (UM, RS, CP, MR). Those components are integrated into an open web-service architecture to support adapted and accessible interaction. The aim is twofold, from the user viewpoint to guarantee personalized students' accessibility needs at HE Institutions, and from the technological perspective to provide open interfaces and web services along with the pervasive use of standards across the architecture and its components, which is meant to support the reusability and extensibility of developments. This has led to the instantiation of the framework and services in different institutions in size, technical and institutional requirements, such as UPV and UNED. 
In addition to developed components, EU4ALL offers a whole framework, including ontologies on user requirements (90 classes modelled and covering 360 instances of services present in the participating institutions), guidelines, handbook of processes and steps to be followed for further implementations [Boticario, 11]. Training, organizational and technical consultancy, are key services offered to adopters willing to improve the accessibility of their Lifelong Learning programmes. Currently, there is no other existing framework focused on accessibility to support interactions validated with real services at large-scale. This is the innovation of EU4ALL beyond the current state of the art and it has been a key project objective from the outset.

There have been contributions to standardisation bodies (ISO, IMS, and W3C), including the different options the project has taking regarding collaboration and contributions from different project partners. The impact for the lack of the ISO bindings (PNP \& DRD) affected the directly involved components in different ways and finally the project produced a XML-binding for the ISO DRD and ISO PNP standards. Actually, EU4ALL has recently served as the main reference for building the new IMS Access for All 3.0. Thus the project has largely fulfilled its objectives with respect to further contribution to the development of the Accessibility Metadata Standards.

Furthermore, the applicability of the EU4ALL approach has been tested both at the two large universities with the highest numbers of students with disabilities in Europe (around 10,000), which were involved in the project from the beginning (i.e. Open University and UNED) as well as in two medium size universities, which join the project in the last year to validate its adoption (IPL and UPV). In this paper, we have reported its applicability at the largest project evaluation site UNED (designer) and a medium-sized univerisity UPV (adopter). Taking into account the variety of issues involved in these two sites the results of the evaluation described in the paper serve to illustrate the coverage of both the approach and developments.

From the point of view of the adoption of the framework, both institutions now must face research transfer processes, from which more stable and scalable framework components are obtained. On the other hand, the interest to adopt EU4ALL components and services varies among different university departments. Interestingly, those services handling information closely related to the ISO/IEC 24751 standard, namely the Disabled Student Services and the Library, have shown more interest in implementing specific EU4ALL components (User Model and Metadata Repository, respectively). Furthermore, replacement of some existing proprietary systems at universities with other open, more standardised solutions is also a required step for the implementation of the framework.

Another question related to the sustainability of the EU4ALL framework is that forthcoming releases of those LMS implementing the EU4ALL framework (Sakai and dotLRN, in the case of the UPV and UNED pilots) take on board the required features to interoperate with the framework.

As for lessons learnt, with respect to the project as a whole some of the main issues are the following: standards-based components and web-based open service architecture approaches have been essential to deal with the wide variety of services that were envisioned in the user requirement analysis and the general and flexible scope of the expected results (i.e. a general framework and architecture to deal with 
the development of services focused on accessible and adaptive learning at HE). Further, thanks to them partners with quite different needs (both from the user and technical viewpoints) and various LMS (i.e. dotLRN, Moodle, Sakai) even with different versions (e.g. two Moodle versions) have been successfully incorporated in the last stage of the project. Similarly, new services and LMS could be implemented in other settings. For instance, in the case of the UPV pilot site, the aforementioned technical outcomes have shown the potential of integrating EU4ALL services into the Sakai framework used at UPV. In other words, the pilot site at UPV has shown the possibility of integrating the EU4ALL framework in an institution that was not involved in the architecture design.

Moreover, the large-scale evaluation outcomes, where students with visual, hearing or physical impairments took part along with all the required stakeholders (lecturers, disability unit officers, technical staff, librarians, content and multimedia officers, etc.) have pointed out specific lessons learnt to be considered from the technical and exploitation viewpoints. The ability of managing multi-format adaptations was greatly valued and not solely for accessibility purposes but for its possibilities to support different types of student with diverse needs. In this and other services the project has gone beyond the current state of services provided to students and service providers (e.g. provide adaptive interactions to contents depending on users profiles) and this has caused mixed reactions on applicability issues depending on the management, technical or end user nature of the stakeholder (e.g. resources required to support the required adaptations). The project showed the need to consider disability officers' support to improve the usage of some services (e.g. NAS). There were concerns related to the costs and management issues required for deploying some services. This reflects an interesting exploitation issue that may be related to the social innovation that this project could leverage.

From the evaluation viewpoint small-scale experiences provided some lessons learnt such as, in the case of UPV, the required assistance by the CEDAT disability office in these experiences and the need to maintain a strong joint between the technical staff and the instructor team. The project experience was globally very positive despite the difficulties of dealing with ongoing developments in their prototype stage and UPV staff consider that the participation in such a large project provided them an important background in order to get involved in projects with multiple partners who belong to different institutions and organizations. Moreover, the exchange of ideas about the EU4ALL potentials also raised very interesting proposals related to accessibility issues which could be addressed in future projects.

Thanks to all the above issues it is expected that the EU4ALL framework, which has succeeded in implementing a package of technical components and services [EU4ALL] that has helped very different $\mathrm{HE}$ institutions to increase their accessibility, could be used as a theoretical and practical guide on how to develop an ICT-supported accessible university (e..g the methodology includes assessments on the level of institution preparedness regarding accessibility practice), which are appreciated for all students, not just those with functional diversity issues: Moreover, the results from this project can also be used as input for the development of personal learning environments (PLE), which help learners take control of and manage their own learning [Van Harmelen, 08]. 


\section{Acknowledgements}

The authors would like to thank the European Commission for the financial support of the EU4ALL project (IST-2006-034478). The work at aDeNu is also supported by the Spanish Ministry of Science and Innovation (TIN2008-06862-C04-01/TSI “A2UN@”). Authors would also like to thank all the EU4ALL partners for their collaboration.

\section{References}

[BenToWeb] Benchmarking Tools for the Web. Available at: http://www.bentoweb.org.

[Beyer, 07] Beyer, S., Meek, A., Kilsby, M., Perry, J. The TATE [Through Assistive Technology to Employment] Project: Outcomes of the TATE Project for people with learning disabilities and staff. Journal of Assistive Technologies, Vol 2, Issue 3, pp. 2-65, 2007.

[Boticario, 06] Boticario, J.G., Cooper, C., Montandon, L., van Dorp, K.J. Towards an Open, Standard-based, Reusable and Extensible Architecture of Services for Accessible Lifelong Learning: An Introduction to the EU4ALL Project. EADTU Annual Conference: Widening participation and opportunities by e-learning in Higher Education. Estonia, November 2006.

[Boticario, 07] Boticario, J.G., Santos, O.C. An open IMS-based user modelling approach for developing adaptive learning management systems. Journal of Interactive Media in Education. Adaptation and IMS Learning Design. Special Issue, 2007/02, pp. 1-19

[Boticario, 08] Boticario, J.G., Santos, O. A standards-based modelling approach for dynamic generation of adaptive learning scenarios. Journal of Universal Computer Science, vol. 14, no. 17, pp. 2859-2876, 2008.

[Boticario, 11] Boticario, J.G, Montandon, K., Oral, M. Publishable Final Activity Report. EU4ALL project, 2011.

[Brusilovsky, 03] Brusilovsky, P., Vassileva,J. Course sequencing techniques for large-scale webbased education. I. J. Cont. Engineering Education \& Lifelong Learning, Vol. 13, Nos.1/2, 2003.

[Buendía, 11] Buendía, F., Roldán, D. Mengod, R., Giménez, T. Making accessible virtual learning environments and their contents, IADIS International E-Society Conference, 2011.

[Carroll, 00] Carroll, J. M. (2000) Making Use: scenario-based design of human-computer interactions. MIT Press, Cambridge, MA.

[Conlan, 02] Conlan, O., Hockemeyer, C., Wade, V., \& Albert, D. Metadata Driven Approaches to Facilitate Adaptivity in Personalized eLearning systems. The Journal of Information and Systems in Education, 1, 38-44, 2002.

[Cudd, 94] Cudd, P.A., Freeman, M., Yates, R.B., Wilson, A.J., Cooke, M.P., Hawley, M.S. The CORES project. Proceedings of the 4th international conference on Computers for handicapped persons table of contents. Springer-Verlag New York, Inc, pp. 493-495, 1994.

[De Bra, 08] Paul De Bra - Adaptive Hypermedia, In: Handbook on Information Technologies for Education and Training, eds. Kinshuk, Sampson, Adelsberger and Pawlowski, pp. 29-46, Springer Verlag, 2008.

[De Bra, 10] De Bra, P., Smits, D., van der Sluijs, K., Cristea A.I., Hendrix, M., GRAPPLE: Personalization and Adaptation in Learning Management Systems, World Conference on 
Educational Multimedia, Hypermedia \& Telecommunications (ED-MEDIA 2010), June 28July 2, 2010, Toronto, Canada.

[Douce, 10] Douce, Christopher; Porch, Wendy and Cooper, Martyn. Adapting e-learning and learning services for people with disabilities. In: 1st International AEGIS Conference: Access for All in the Desktop, Web and Mobile Field: an End-user and Developer Perspective, 7-8 October 2010, Seville, Spain.

[Dunn, 03] Sarah Dunn. Return to SENDA? Implementing accessibility for disabled students in virtual learning environments in UK further and higher education. City University, 2003. Retrieved on January 2011 from: http://www.saradunn.net/LMSreport/

[Edutools] Edutools. Available at: http://www.edutools.info/index.jsp?pj=1

[E-Framework] E-Framework. Available at: http://www.e-framework.org

[EC-TEL, 10] Fifth European Conference on Technology Enhanced Learning | Sustaining TEL: from innovation to learning and practice, 2010. Available at: http://www.ectel2010.org/component/content/frontpage

[EHEA, 09]. The European Higher Education Area in a global context. Report on overall developments at the European, national and institutional levels. Approved by BFUG at its meeting in Prague, 12-13 February 2009. Retrieved on July 2011 from: http://www.ond.vlaanderen.be/hogeronderwijs/bologna/conference/documents/2009_EHEA_in _global_context.pdf

[EU-Riga, 06] Ministers of the European Union (EU) Member States and accession and candidate countries, European Free Trade Area (EFTA) countries and other countries adopted a Declaration on eInclusion (Riga, 11-13 June 2006).

[EU4ALL] EU4ALL website: http://www.eu4all-project.eu/

[eXe] eXe. Available at: http://exelearning.org

[Felder, 88] Felder R. M. and Silverman L. K. Learning and Teaching Styles In Engineering Education, Engr. Education, 78(7), p. 674-681, 1988.

[Fink, 98] Fink, J., Kobsa, Nill, A. Adaptable and Adaptive Information Provision for All Users, Including Disabled and Elderly People, 1998. Retrieved April 15, 2011, from: http://www.isr.uci.edu/ kobsa/papers/1998-NRHM-kobsa.pdf

[Fernández del Viso, 11] Fernández del Viso, A., Vázquez de Prada, E. D6.2.6 Revision of the technical validation, user evaluation and programme evaluation plans, EU4ALL project, 2011.

[FLEXO] FLEXO project. Available at: http://www.ines.org.es/flexo

[Fluid] Fluid project. Available at: http://fluidproject.org

[Ghali, 08] Ghali, F. and Cristea, A. Interoperability between MOT and LMS: Converting CAF to IMS QTI and IMS CP, AH 2008: 5th Int. Conf. on Adaptive Hypermedia and Adaptive Web-Based Systems. Berlin Heidelberg, Springer, p. 296-299, 2008.

[Hauger, 07] Hauger, D. and Kock, M. State of the Art of Adaptivity in E-Learning Platforms. Workshop at Adaptivity and User Modeling in Interactive Systems ABIS 2007.

[HEAG] Higher Education Accessibility Guidelines. Available at: http://www.heagnet.org

[Herrera, 09] Herrera Tirado, V., González Morcillo, C., Lacave Rodero, M.C. An Educational Tool for People with Hearing Disabilities Based on Bayesian Networks. EDULEARN 2009.

[IMS-BLTI] http://www.imsglobal.org/lti/blti/bltiv1p0/ltiBLTIimgv1p0.html 
[ISO, 09] ISO/IEC 24751 Individualized adaptability and accessibility in e-learning, education and training, 2009.

[Karagiannidis, 00] Karagiannidis, C. and Sampson, D.G. (2000) Layered Evaluation of Adaptive Applications and Services. International Conference on Adaptive Hypermedia and Adaptive Web-Based Systems: AH2000, Trento (Italy), Springer-Verlag Berlin Heidelberg.

[Lanzilotti, 06] Lanzilotti, R., Ardito, C., Costabile, M. F. and Angeli, A. D. (2006) eLSE Methodology: a Systematic Approach to the e-Learning Systems Evaluation. Educational Technology and Society, 9 (4), p. 42-53.

[LOM] IEEE Learning Object Metadata. Available at http://ltsc.ieee.org/wg12/20020612-FinalLOM-Draft.html.

[LTSA] IEEE Learning Technology Systems Architecture Available at: http://ltsc.ieee.org/wg1/files/ltsa-400.html.

[May, 10] May, H. and Bridger, K. Developing and embedding inclusive policy and practice in higher education. The Higher Education Academy, 2001 Retrieved on December 2010 from: http://www.heacademy.ac.uk/assets/York/documents/ourwork/inclusion/DevelopingEmbedding InclusivePP_doc.doc, 2010.

[MICOLE] Multimodal Collaboration Environment for Inclusion of Visually Impaired Children. Available at: http://micole.cs.uta.fi/

[Moreno, 09] Moreno, G., Martínez, L., Boticario, J.G., Fabregat, R. Research on Standards Supporting A2UN@: Adaptation and Accessibility for ALL in Higher Education. Proceedings 3rd Workshop Towards User Modeling and Adaptive Systems for All (TUMAS-A 2009).

[Muñoz-Merino, 09] Muñoz-Merino, P.J., Delgado-Kloos, C. and Fernández-Naranjo, J. Enabling Interoperability for LMS Educational Services. Computer Standards and Interfaces, vol. 31:2, p. 484-498, 2009.

[Muñoz-Organero, 10] Muñoz-Oganero, M., Muñoz-Merino, P.J., Delgado Klos, C., Personalized Service-Oriented E-Learning Environments. IEEE Internet Computing, MarchApril, 2010.

[OKI] Open Knowledge Initiative. Available at: http://www.okiproject.org

[Petrie, 09] Petrie, H., Power, C., Swallow, D. . D1.1.3 - Report on ontology of users and their requirements. EU4ALL project, 2009.

[Phipps, 06] Phipps, L. and Kelly, B. Holistic Approaches to E-Learning Accessibility, In: ALT-J: Research In Learning Technology, Vol. 14, No. 1, March, p. 69-78.

[Raffenne, 09] Raffenne, E., Santos, O.C., Granado, J., Couchet, J., and J.G. Boticario. Integrating OpenACS/dotLRN with an Open and Accessible Service Architecture Framework. $8^{\text {th }}$ OpenACS/.LRN Conference, Costa Rica, 2009.

[Roldán, 11] Roldán, D., Mengod, R., Merino, D, Sakai. Administración, configuración y desarrollo de aplicaciones. Ed. RA-MA, 2011.

[Rodríguez-Ascaso, 08] Rodríguez-Ascaso, A., L. Martin, E. Gutiérrez y Restrepo, C. Finat, O. C. Santos, J. G. Boticario, "Design and Use of an Online Community for Students with Disabilities. The UNED Experience", EADTU's 20th Annual Conference, France, 2008.

[Rodríguez, 02] Rodríguez, O., Chen, S., Shi, H., and Shang, Y. Open Learning Objects: the case for inner metadata. In Proceedings of the 11th World Wide Web Conference (WWW2002) (Alternate Track on Education), Honolulu, HI, 2002. 
[Santos, 07] Santos, O.C., Boticario, J.G., Raffenne, E. and Pastor, R. Why using dotLRN? UNED use cases. In J.R. Rodríguez and M. Palomo (Eds.) Proceedings of the FLOSS (Free/Libre/Open Source Systems) International Conference 2007, UCA Pub, p. 195-212, 2007.

[Santos, 10] Santos, O. C., C. Barrera, E. Mazzone, J. G. Boticario, Catering for personal autonomy with e-mentoring supported by recommendations. Learning Design: Handbook of Research on Personal Autonomy Technologies and Disability Informatics, United Kingdom, Information Science Reference, IGI Global, 2010.

[Santos, 11a] Santos, O.C., Boticario, J.G., Raffenne, E., Granado, J., Rodriguez-Ascaso, A., Gutierrez y Restrepo, E. A standard-based framework to support personalisation/adaptation and interoperability in inclusive learning scenarios. In Fotis Lazarinis, Steve Green, Elaine Pearson (Eds.) Handbook of Research on E-Learning Standards and Interoperability: Frameworks and Issues, IGI Global, pp 126-169, 2011.

[Santos, 11b] Santos, O.C. and Boticario, J.G. Requirements for Semantic Educational Recommender Systems in formal e-learning scenarios. Algorithms 4 (2), pp. 131-154, 2011.

[Santos, 11c] Santos, O.C., Boticario, J.G. A General Framework for Inclusive Lifelong Learning in Higher Education Institutions with Adaptive Web-Based Services that Support Standards. In E-Infrastructures and Technologies for Lifelong Learning: Next Generation Environments, IGI Global, pp 29-58, 2011

[Sawyer, 08] Sawyer, R.K. Optimizing learning: Implications of learning sciences research. In: Innovating to learn, learning to innovate. Paris, OECD, 2008.

[SCORM] Sharable Content Object Reference Model. Available at: http://www.socorm.com

[Seale, 06] Seale, J., E-learning and disability in higher education: accessibility research and practice. Routledge London, 2006.

[Seale, 08] Seale, J., Draffan, E.A and Wald, M. Exploring disabled learners' experiences of elearning: LEXDIS Project Report. Southampton, UK, University of Southampton, 2008.

[Seale, 10] Seale, J. and Cooper, M. E-learning and accessibility: An exploration of the potential role of generic pedagogical tools. In Computers and Education, Volume 54, Issue 4, May 2010, p. 1107-1116, 2010.

[Van Harmelen, 08] Van Harmelen, H. Design trajectories: four experiments in PLE implementation. Interactive Learning Environments, 1744-5191, Vol. 16, Issue 1, 35-46, 2008.

[Van Rosmalen, 04] Van Rosmalen, P., Boticario, J.G. and Santos, O.C. The Full Life Cycle of Adaptation in aLFanet eLearning Environment. Learning Technology newsletter. Vol. 4, p. 5961, 2004.

[Weiermair-Märki, 09] Weiermair-Märki, C., Unterfrauner, E., Kesselring, A., Montandon, L., Arjona, M. D1.4.6 Revised market study on the institutional conditions for the implementation of EU4ALL, 2009.

[White, 05] White, A. Introducing the Sakai Community. 4th Sakai Conference, Austin, TX, USA, 2005.

[WSRP] http://www.oasis-open.org/committees/tc_home.php?wg_abbrev=wsrp 